\title{
Gleichstellungspolitik vor alten und neuen Herausforderungen - Welchen Beitrag leistet die Familienpolitik?
}

Um Gleichstellungspolitik ist es in Deutschland nach dem Scheitern des Gleichstellungsgesetzes für die Privatwirtschaft ruhig geworden. Aktuelle Diskussionen drehen sich um Familienpolitik, die mit der Orientierung auf die bessere Vereinbarkeit von Familie und Beruf ein altes gleichstellungspolitisches Thema aufgegriffen hat. Braucht Deutschland eine Gleichstellungspolitik, die darüber hinausgeht? Ist die Lösung des Vereinbarkeitsproblems gegenwärtig die wichtigste Frage? Welchen Beitrag kann die neue Familienpolitik zur Gleichstellung von Frauen und Männern leisten?

\section{Problemstellung}

Auf bundespolitischer Ebene hat es in den letzten Jahren fast keine gleichstellungspolitischen Initiativen mehr gegeben. Familienpolitik hat dagegen einen großen Aufschwung erlebt. Rechtfertigt der Stand der Gleichstellung von Frauen und Männern, Gleichstellungspolitik zurückzustellen? Auf Basis einer knappen Analyse wird im Abschnitt 2 die Gleichstellungssituation in Deutschland eingeschätzt: Wo hat es Fortschritte gegeben, wo zeigen sich Stagnationstendenzen oder gar Rückschritte? Ich werde im Rahmen dieser Betrachtung begründen, dass eine Gleichstellungspolitik, die über Vereinbarkeitsförderung hinausgeht, erforderlich ist, um die soziale Ungleichheit von Frauen und Männern zu überwinden.

Abschnitt 3 behandelt Entwicklungen, die die Gleichstellungspolitik vor neue Herausforderungen stellen. Ökonomische Umbrüche, die auch eine zunehmende Differenzierung innerhalb der Genusgruppen mit sich bringen, erschweren eine solidarische Politik, die sich an der Geschlechterfrage orientiert. Infolge der wachsenden sozialen Ungleichheit stellt sich die Geschlechterfrage in modifizierter Weise: Verschiedene ungleichheitsrelevante Dimensionen überlagern sich und machen differenzierte Politikkonzepte erforderlich.

Anschließend (Abschnitt 4) wird die aktuelle Familienpolitik unter die Lupe genommen. Ihr Ziel, die Vereinbarkeit von Familie und Beruf zu verbessern, ist seit Langem auch ein gleichstellungspolitisches Anliegen. Nun wurden oft geforderte Ver- besserungen in Gesetzesform gegossen (Tagesbetreuungsausbaugesetz, Elterngeld). Weitere Projekte sind mit dem Ausbau von Ganztagsschulen und zusätzlichen Krippenplätzen anvisiert. Dass die neue Familienpolitik geschlechterpolitisch nicht neutral ist, lässt schon der Widerstand konservativer Kreise, beispielsweise gegen die „Vätermonate“ beim Elterngeld, vermuten. Frauenpolitikerinnen sehen dagegen die Gefahr, dass gleichstellungspolitische Anliegen durch die Fokussierung auf das Vereinbarkeitsthema verdrängt werden. Wo liegen die Potenziale, aber auch die Grenzen der Familienpolitik in gleichstellungspolitischer Hinsicht? Das Fazit (Abschnitt 5) fasst Schnittmengen beider Politiken zusammen und unterstreicht den Bedarf an umfassenden Gleichstellungsansätzen.

\section{Gleichstellung der Geschlechter in Deutschland}

\subsection{ANGLEICHUNGSPROZESSE}

Unzweifelhaft haben in den letzten Jahrzehnten Veränderungen in den Geschlechterverhältnissen in Deutschland stattgefunden. Wer nur darauf schaut, läuft allerdings Gefahr, die Fortschritte in Bezug auf die Gleichstellung von Frauen und Männern zu überschätzen, auch wenn die Angleichungsprozesse trotz ihrer Begrenztheit für die Chancen von Frauen auf ein gleichberechtigtes Leben bedeutsam sind. Wesentliche Fortschritte in Bezug auf die Gleichstellung von Frauen und Männern hat es vor allem in Bezug auf die rechtliche
Gleichstellung, bei der Bildung, in den Einstellungen zu Geschlechterrollen sowie in der politischen Repräsentation gegeben:

(1) Die rechtliche Gleichstellung von Frauen und Männern ist in Deutschland weitgehend vollzogen (zur Entwicklung vgl. Berghahn 2003). Darüber hinaus bestehen gesetzliche Regelungen für einzelne Bundesländer oder Behörden, die auf die Förderung von Frauen zielen, um historisch überkommene Nachteile zu kompensieren. Beispiele dafür sind das Bundesgleichberechtigungsgesetz und die Gleichstellungsgesetze einiger Bundesländer. Vergleichbare Gesetze gibt es für die private Wirtschaft nicht. Auch darf das Geschlecht nach dem Allgemeinen Gleichbehandlungsgesetz (AGG) kein Grund für Ungleichbehandlung sein.

(2) Die traditionelle Ungleichheit im Bildungsniveau von Frauen und Männern macht sich heute fast nur noch bei den älteren Kohorten bemerkbar. Bei den schulischen Abschlüssen haben Frauen Männer sogar überholt, bei den beruflichen Abschlüssen hat sich das Niveau immer weiter angeglichen. So hatten 2004 knapp $50 \%$ der Frauen gegenüber gut 51 \% der Männer eine Lehr- oder Anlernausbildung. Beim Fach-/Hochschulabschluss liegen Frauen zwischen 30 und 35 Jahren noch

Christina Klenner, Dr., Wissenschaftlerin im WSI in der Hans-Böckler-Stiftung. Arbeitsschwerpunkte: Frauenerwerbstätigkeit, Geschlechterverhältnisse, Work-Life Balance.

e-mail: christina-klenner@boeckler.de 
unter dem Anteil der Männer, die unter 30jährigen Frauen haben dagegen auch hinsichtlich dieses Kriteriums die Männer überholt (Bothfeld et al. 2005, S. 67).

(3) Die Einstellungen zu den Geschlechterrollen haben sich modernisiert. Die traditionelle Arbeitsteilung der Geschlechter befürworteten 2004 nur noch $40 \%$ der Westdeutschen gegenüber $70 \%$ im Jahr 1982, und nur $17 \%$ der Ostdeutschen (Statistisches Bundesamt 2006, S. 520). Junge Frauen können sich heute selbstverständlicher für unterschiedliche Lebenswege entscheiden. Die Präsentation des traditionellen Frauenbildes in Medien und Öffentlichkeit hat sich abgeschwächt. Jedoch sind die Fortschritte mit Rückschritten gepaart, traditionelle Bilder sind teilweise wieder auf dem Vormarsch.

(4) Die politische Repräsentanz von Frauen hat sich - insbesondere seit der Einführung von Quotenregelungen bei einigen Parteien und Gewerkschaften - deutlich verbessert. Der Anteil von Frauen im deutschen Bundestag hat sich langfristig erhöht, auch wenn er in der gegenwärtigen Legislaturperiode auf 31,6 \% geringfügig abgesunken ist. Mit einem Frauenanteil von $37,5 \%$ in der Regierung liegt Deutschland hinter den skandinavischen Ländern auf einem der vorderen Plätze in Europa.

Diesen positiven Veränderungen stehen äußerst zähe, langsame Entwicklungen, Stagnationsprozesse und teilweise Rückschritte in anderen entscheidenden Bereichen gegenüber. Vor allem materielle Ressourcen und ökonomische Macht sind noch extrem ungleich verteilt, wie im nächsten Abschnitt gezeigt wird. Den meisten Frauen ist das auch bewusst (Institut für Demoskopie Allensbach 2006). ${ }^{1}$

\subsection{WIDERSPRÜCHLICHE FORTSCHRITTE, STAGNATION, RÜCKSCHRITTE}

Ich werde mich im Folgenden auf Gleichstellung im Erwerbsleben konzentrieren. Gleichstellung in diesem Bereich ist ein Kernelement der Überwindung sozialer Ungleichheit, da in Industriegesellschaften der Erwerb des Lebensunterhalts, die soziale Sicherung, aber auch die gesellschaftliche Integration wesentlich über Erwerbsarbeit erfolgt. Daneben sind für eine umfassende Einschätzung des Standes der
Gleichstellung natürlich weitere Fragen, wie etwa Absicherung von sozialen Risiken, Gewalt gegen Frauen, Frauengesundheit, bedeutungsvoll.

\section{ERWERBSTÄTIGKEIT: ZUNAHME MIT PROBLEMEN}

Obwohl Frauen den Bildungsvorsprung der Männer weitgehend aufgeholt haben, können sie ihre Bildungserfolge ${ }^{2}$ nur unzureichend in entsprechende Erwerbspositionen umsetzen. Das hat qualitative und quantitative Seiten. So ist zwar einerseits die Zahl der erwerbstätigen Frauen stetig gestiegen, die Erwerbstätigenquote beträgt nun für Frauen 59,5 \% (2005, Mikrozensus). Der geschlechtsspezifische Unterschied ist immer noch groß, aber im Laufe der Jahre geringer geworden.

Einige Probleme kommen in der steigenden Quote nicht zum Ausdruck. So verdeckt die gesamtdeutsche Zahl, dass die Erwerbstätigenquote der Frauen im Westen angestiegen, im Osten entgegen dem gesamtdeutschen Trend aber zurückgegangen ist. Frauen wären hier gern viel häufiger beruflich tätig. Das Gleiche gilt für Mütter kleiner Kinder. Mangelnde Kinderbetreuungsmöglichkeiten und unpassende Arbeitszeiten führen dazu, dass ein großer Teil der Mütter entgegen ihren Wünschen einige Jahre nach der Geburt eines Kindes nicht erwerbstätig ist (OECD 2001).

Darüber hinaus ist die Erwerbstätigenquote ein unzureichender Gradmesser, um die Teilhabe von Frauen an Erwerbsarbeit zu messen (Beckmann 2003; Rüling in diesem Heft). Die Dauer der Arbeitszeit muss ebenfalls einbezogen werden. Das Arbeitsvolumen von Frauen ist seit 1991 nicht gewachsen, weil eine steigende Zahl von Frauen ein sogar leicht gesunkenes Arbeitsvolumen unter sich aufteilt (Wanger 2005). Die Zunahme der Frauenerwerbstätigkeit entpuppt sich zu einem Teil als Zunahme von nicht existenzsichernder Teilzeitarbeit einschließlich Minijobs. Das Arbeitsvolumen ist gleichstellungspolitisch nicht ohne Bedeutung: Im internationalen Vergleich geht ein geringer Anteil von Frauen am gesamtwirtschaftlichen Arbeitszeitvolumen mit einer geringen Zahl weiblicher Führungskräfte einher und umgekehrt (Funke/Suder 2007).

Auch die qualitative Seite der Arbeit lässt häufig zu wünschen übrig. Die geschlechtsspezifische Segregation konnte so gut wie nicht abgebaut werden (EU-Kom- mission 2007). Frauen sind nicht selten in beruflichen Sackgassen beschäftigt. Ein Teil der Beschäftigungsverhältnisse ist prekär (Abschnitt 3.2).

\section{EINKOMMENSUNTERSCHIEDE GRAVIEREND}

Der geschlechtsspezifische Einkommensabstand ("gender pay gap") hat sich seit Beginn der 1990er Jahre nur noch langsam um 0,6 bis 6 Prozentpunkte je nach zugrundegelegter Datenquelle - verringert (Bothfeld et al. 2005, S. 262f.). In Ostdeutschland, wo der geschlechtsspezifische Abstand geringer ist, stagniert die Entwicklung. Besonders problematisch ist, dass der Einkommensunterschied zwischen Frauen und Männern gerade dort am höchsten ist, wo die Beschäftigtenanteile zunehmen: bei den Angestellten, darunter bei den höher Qualifizierten. Dadurch ist der gesamtdeutsche Durchschnitt insgesamt im europäischen Vergleich auch sehr hoch (Abbildung 1). Er beträgt 22 bis $26 \%$ (je nach Quelle: EUKommission 2007; Jurczak/Hurley 2007).

Im gender pay gap kommen mehrere Faktoren zusammengefasst zum Ausdruck. Die sich kaum verändernde geschlechtsspezifische Segregation, das heißt die Verteilung von Frauen und Männern auf Branchen und Tätigkeiten, wirkt sich stark zulasten der durchschnittlichen Fraueneinkommen aus: Frauen arbeiten überwiegend dort, wo weniger verdient wird (Bothfeld et al. 2005, S. 256ff.). Auch das Tarifsystem ist mit den zugrunde liegenden Bewertungen von Arbeitstätigkeiten nicht geschlechtsneutral (Bundesregierung 2002). „Frauentypische“ Lebensläufe mit familienbedingten Erwerbsausstiegen, Teilzeitphasen und nicht qualifikationsgerechter beruflicher Tätigkeit sind mit großen Einbußen im Lebenserwerbseinkommen verbunden (Klammer 2007).

Daneben wirkt sich die vertikale Segregation einkommensmindernd für Frauen aus. Frauen sind häufig auf den unteren und mittleren Ebenen der betrieblichen

\footnotetext{
Nur $28 \%$ der Frauen, aber 49 \% der Männer halten die Gleichberechtigung weitgehend für verwirklicht.

2 Zugleich gibt es im Bereich der Bildung erhebliche Probleme, wie den mangelnden Zugang von Frauen zu Ausbildungsplätzen, die geschlechtsspezifischen Ausbildungswege (duales System und vollzeitschulische Ausbildungen), auf die hier nicht eingegangen werden kann.
} 
Abb. 1: Geschlechtsspezifische Einkommenslücke EU-27, 2005 Differenz zwischen durchschnittlichen Fraueneinkommen und Männereinkommen in \% des durchschnittlichen Männereinkommens

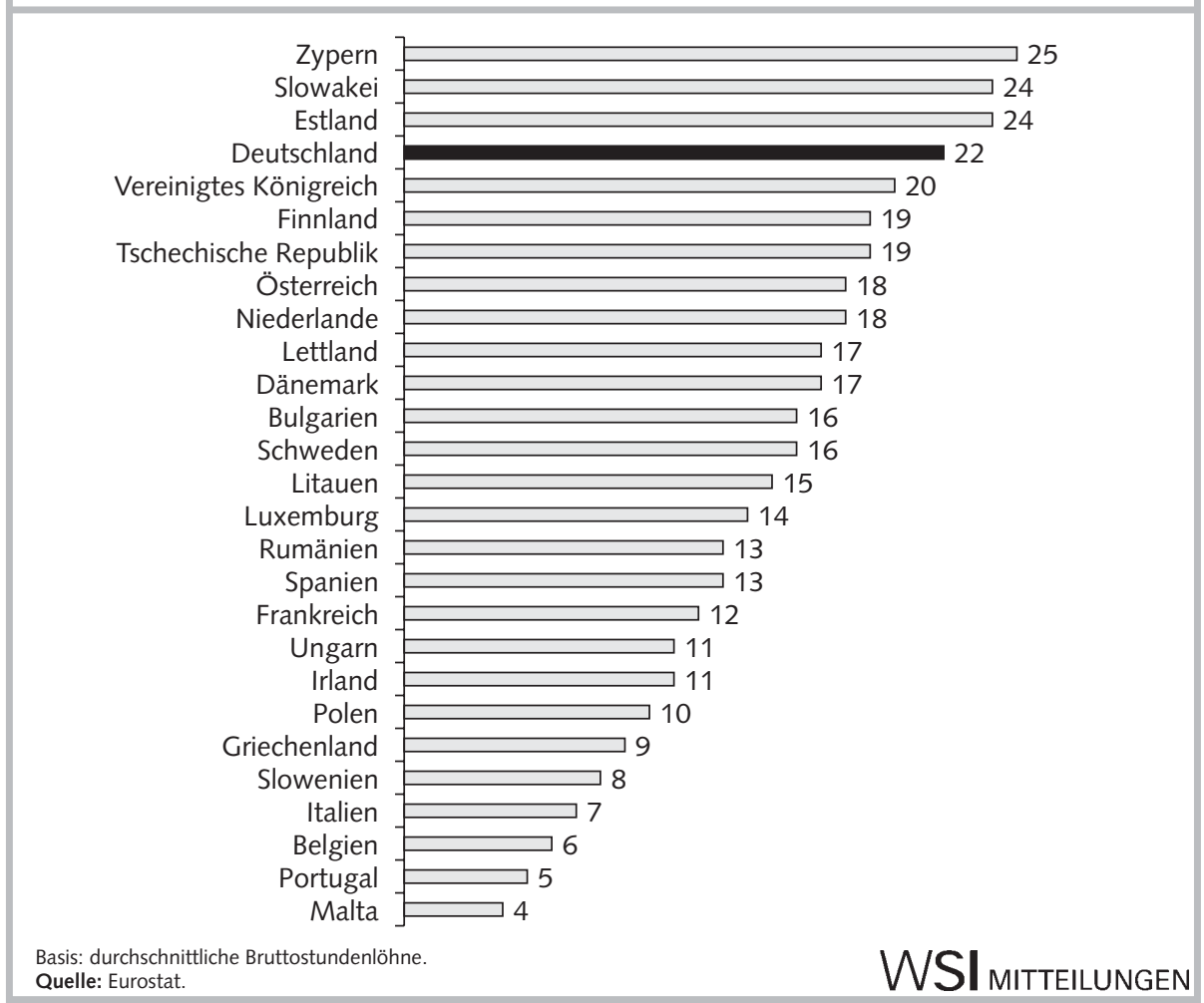

und damit auch Einkommenshierarchie tätig. Bis in Spitzenpositionen gelangen sie nur selten (Bender/Lewerenz 2006; Holst/ Schrooten 2006; Holst/Stahn 2007; Bundesregierung 2006). Die höchsten Positionen in der Wirtschaft sind fast frauenfrei.

Da diese strukturellen Faktoren nicht den gesamten geschlechtsspezifischen Unterschied erklären, muss auch auf eine Einkommensdiskriminierung ${ }^{3}$ von Frauen geschlossen werden. Im Durchschnitt erhalten Frauen auch im selben Beruf $22 \%$ weniger als ihre männlichen Kollegen (WSI-Frauenlohnspiegel). ${ }^{4}$

\section{UNTERSCHIEDE IN DEN ARBEITS- ZEITEN VERGRÖßERN SICH}

Bei den durchschnittlichen Arbeitszeiten ist die Schere zwischen den Geschlechtern in den letzten Jahren weiter auseinandergegangen und beträgt mehr als zehn Stunden pro Woche: Erwerbstätige Männer arbeiten knapp 41, Frauen rund 30 Stunden durchschnittlich pro Woche (2005). ${ }^{5}$ Zwar sind dafür heute mehr Frauen überhaupt erwerbstätig; ein Teil von ihnen würde ohne Teilzeitarbeit, mit der Familie und Beruf vereinbart wird, eventuell ganz außerhalb des Arbeitsmarktes stehen.
Auch wenn der Anspruch auf Teilzeitarbeit nach dem Teilzeit- und Befristungsgesetz 2001 im Sinne erweiterter Optionalität für abhängig Beschäftigte sinnvoll ist, ist doch die Zunahme der Teilzeitarbeit als eine von Frauen dominierte Form der Erwerbstätigkeit keineswegs unproblematisch für deren Gleichstellung. Mit Teilzeitarbeit sind nicht nur von der absoluten Höhe her meist nicht existenzsichernde Einkünfte verbunden. Auch relativ - umgerechnet auf Stundenlöhne sind Teilzeitbeschäftigte und Minijober überproportional von niedrigen Löhnen betroffen (Weinkopf/Kalina 2006). Teilzeitbeschäftigte verdienen durchschnittlich $4 €$ weniger pro Stunde als Vollzeitbeschäftigte (StBA 2007). Ob hierfür nur strukturelle Effekte verantwortlich sind etwa, in welchen Segmenten überhaupt Teilzeitarbeit angeboten wird, - oder inwieweit Teilzeitbeschäftigte diskriminiert werden, ist bislang unzureichend erforscht.

Problematisch für die Gleichstellung ist ebenfalls, dass Teilzeitarbeit für eine Veränderung der innerfamilialen Arbeitsteilung wenig Chancen bietet: In Paarhaushalten mit Teilzeit beschäftigten Frauen haben Männer keine weiteren Anteile an der Fa- milienarbeit übernommen (Rüling in diesem Heft; Kratzer et al. 2005).

Statt der von frauenpolitischer Seite geforderten Arbeitszeitverkürzung für alle, die lange Zeit als Schlüssel zu einer gleichberechtigten Teilhabe von Frauen am Erwerbsleben und von Männern an der Familie angesehen wurde, ist die geschlechtsspezifische Polarisierung der Arbeitszeiten immer größer geworden. Die durchschnittlichen Arbeitszeiten der Männer steigen wieder an, die Arbeitszeiten von Frauen gehen im Durchschnitt immer weiter zurück (Bothfeld et al. 2005). Von geschlechtergerechten Arbeitszeiten im Rahmen eines Zweiverdienermodells kann bisher keine Rede sein (Klenner/Pfahl 2007).

Dabei ist die Verbreitung von Teilzeitarbeit keineswegs nur auf Vereinbarkeitsprobleme zurückzuführen, sie wird vielmehr von Arbeitgebern auch zur Kostenreduktion und Arbeitszeitflexibilisierung eingesetzt. Viele Teilzeitbeschäftigte arbeiten weniger Stunden als gewünscht. Der geschlechtsspezifische Unterschied in den Arbeitszeiten wäre bei Weitem nicht so groß, wenn die tatsächlichen Arbeitszeiten den Arbeitszeitwünschen entsprechen würden (Holst 2007). Ein beträchtlicher Teil der Teilzeitarbeit (in Ostdeutschland rund die Hälfte) ist gänzlich unfreiwillig.

\subsection{NOCH LÄNGST KEINE GLEICHSTELLUNG - HANDLUNGSBEDARF BLEIBT}

Der knappe Überblick zeigt, dass der Stand der Gleichstellung von Frauen und Männern keineswegs rechtfertigt, Gleichstellungspolitik zurückzustellen. Eine Angleichung lässt sich vorwiegend im politischen und rechtlichen Bereich und im Denken konstatieren. Angleichungsprozesse im Bereich der Erwerbsarbeit vollziehen sich nur langsam und sind widersprüchlich. Das zeigt sich beispielsweise am Wachstum der

\footnotetext{
3 Je nach zugrunde gelegter Datenquelle liegt der Diskriminierungsanteil am Einkommensunterschied in Westdeutschland bei 12 bis $32 \%$, in Ostdeutschland bei 11 bis $24 \%$ (Bothfeld et al. 2005)

$4 \quad$ Im Rahmen des WSI-Lohnspiegels konnten bisher 68.000 Einträge in einer Online-Umfrage ausgewertet werden, vgl. http://www.boeckler.de/cps/ rde/xchg/SID-3D0AB75D-96BC1202/hbs/hs.xsl/ 320 85262.html

5 Normalerweise geleistete Arbeitszeit; Quelle: Mikrozensus.
} 
Frauenerwerbstätigkeit in Beschäftigungssegmenten, in denen schlecht verdient wird, sodass der geschlechtsspezifische Einkommensabstand selbst bei Vollzeit erheblich ist. Es zeigt sich am Anachronismus des faktischen Ausschlusses von Frauen aus höheren ökonomischen Entscheidungsfunktionen.

Widersprüchliche Folgen hat das $\mathrm{Zu}$ sammenfallen von Emanzipationsbestrebungen von Frauen und ökonomischen Interessen. Frauen wollen zunehmend erwerbstätig sein und bekommen doch nicht selten nur einen Minijob oder Teilzeitarbeit mit geringer Stundenzahl, da diese Jobs Personaleinsatzstrategien entgegenkommen. So nimmt zwar die Erwerbstätigkeit von Frauen zu, aber oft in Gestalt nicht existenzsichernder Arbeit. Diese Form der Erwerbsintegration gewährt keine ökonomische Autonomie und sie stellt die traditionelle häusliche Arbeitsteilung nicht infrage.

Der Blick darauf, dass die Erwerbstätigkeit von Frauen steigt, reicht auch keineswegs aus, denn er verbirgt, dass ein nicht geringer Teil der Frauen langzeitarbeitslos ist und durch die Sozialgesetzgebung in Abhängigkeit vom Partner leben muss.

So muss der gleichstellungspolitische Blick nicht nur auf eine steigende Frauenerwerbstätigkeit und verringerte Arbeitslosigkeit gerichtet werden, sondern vor allem auf die Qualität der Erwerbsintegration von Frauen. Das bedeutet vor allem ${ }^{6}$

- die Einkommensfrage zentral zu behandeln: Diskriminierungen aufzudecken und $\mathrm{zu}$ beseitigen und Arbeitstätigkeiten geschlechtergerecht neu zu bewerten,

- die geschlechtsspezifische Segregation der Tätigkeitsfelder - nach Berufen, Branchen, Hierarchieebenen - wieder stärker zu thematisieren und auf ihre Auflösung hinzuwirken,

- ein Konzept für geschlechtergerechte Arbeitszeiten im Rahmen eines Zweiverdienermodells zu entwickeln, dabei die Vorschläge zu lebenslaufbezogenen Arbeitszeiten zu nutzen und auf eine sozialpolitische Absicherung von Lebensphasen zu dringen.

Welche Rolle spielt hierfür eine Politik der besseren Vereinbarkeit von Familie und Beruf? Einige der skizzierten Ungleichgewichte haben auch mit der schwierigen Vereinbarkeit von Familie und Beruf zu tun. Das gilt für die Behinderung der Mütter- erwerbstätigkeit und für die Ausbreitung von Teilzeitarbeit ebenso wie für die Einkommensminderungen durch frauentypische Erwerbslebensverläufe. Doch die mangelnde Vereinbarkeit erklärt bei Weitem nicht alles: Diskriminierung bei den Einkommen, Unterbewertung von typischer Frauenarbeit, die „gläserne Decke“, die Frauen völlig unabhängig vom Vorhandensein von Kindern am Aufstieg hindert, sind Beispiele für Ursachen von Geschlechterungleichheit, denen mit Vereinbarkeitsförderung nicht beizukommen ist. Deshalb ist wichtig, Gleichstellungspolitik mit einem umfassenden Ansatz (wieder) auf die politische Agenda zu setzen. Das gilt auch deshalb, weil sich neue Herausforderungen für Gleichstellungspolitik entwickelt haben.

\section{Neue Herausforderungen
für Gleichstellungspolitik}

In den letzten Jahren sind die Unterschiede zwischen Frauen größer geworden. Das ist zum einen durch die insgesamt zunehmende soziale Ungleichheit in Deutschland bedingt, zum anderen spielen auch Pluralisierungsprozesse eine Rolle. Die Spannweite dessen, was Frauen verdienen, wie lange sie arbeiten und welche beruflichen Chancen sie haben, ist heute beträchtlich (Bothfeld et al. 2005). Verschiedene ungleichheitsrelevante Dimensionen - Klasse und Schicht, Herkunft und Wohnort, darunter auch Ost-West-Differenzen, Alter oder Behinderung - überlagern und verschränken sich in ihrer Wirkung (Gottschall 2000). $\mathrm{Zu}$ den differenzierenden Merkmalen könnte künftig verstärkt das Kriterium gehören, ob Beschäftigte Fürsorgeverantwortung wahrnehmen, wie im nächsten Abschnitt sichtbar wird.

Im Folgenden wird auf Umbruchprozesse in der Arbeit hingewiesen, die gegensätzliche Wirkungen auf verschiedene Gruppen von Frauen haben: Für einige entstehen Chancen, wenn auch riskante, für andere gestalten sich Arbeits- und Lebensbedingungen zunehmend prekär. Das stellt Gleichstellungspolitik vor neue Fragen.

\subsection{RISKANTE CHANCEN FÜR DIE EINEN}

Der „Umbruch in der Arbeit“ (Sauer 2005) geht mit einer „forcierten Vermarktli- chung " der gesellschaftlichen Organisation von Arbeit einher. Damit sind Prozesse umschrieben wie Ausrichtung am Kunden, flexible bedarfsgerechte Personalanpassung, flexible Arbeitszeitorganisation (, atmende Fabrik"), ergebnis- und erfolgsorientierte Leistungspolitik sowie eine Arbeitsorganisation, die „unternehmerisches Handeln" von einzelnen Beschäftigten fordert. Von den Arbeitnehmerinnen und Arbeitnehmern werden Verhaltensweisen gefordert, die dem sogenannten „Arbeitskraftunternehmer" eigen sind, einem Typus, der künftig vermehrt gefragt sein könnte: unter Bedingungen entgrenzter Arbeit kontrolliert und ökonomisiert der Arbeitskraftunternehmer sich selbst, sodass schließlich die gesamte Lebensführung „verbetrieblicht wird“ (Jurczyk/Voß 2000).

Empirische Untersuchungen zeigen, dass „der Arbeitskraftunternehmer deutlich weiblich konnotiert ist" (Voß/Weiß 2005, S. 79). Voß und Weiß beobachten bei qualifizierten weiblichen Angestellten eine auffallende „private und berufliche Reflektiertheit", mit der sie „unterschiedliche, auch konfligierende Ansprüche an ihre Tätigkeit gezielt in eine flexible Berufsstrategie“ (ebd. S. 80) umsetzen. Der auch als „Subjektivierung" der Arbeit beschriebene Neuzuschnitt der Arbeitsanforderungen bedingt offenbar - trotz ökonomischer Zwänge und zunehmender Entgrenzungstendenzen - auch Chancen für Autonomie in der Arbeit und ist für Frauen zum Teil attraktiv.

Angesichts der gewachsenen Qualifikation und der Leistungsbereitschaft von Frauen wollen Betriebe zunehmend auf dieses Potenzial nicht mehr verzichten, allen Geschlechterstereotypen zum Trotz. Ergebnisse qualitativer Forschung deuten darauf hin, dass Frauen in bestimmten beruflichen Segmenten weitgehend ohne direkte Gleichstellungspolitik Chancen auf interessante berufliche Herausforderungen und auch auf beruflichen Aufstieg haben. So könnten sich mit dem Strukturwandel von Arbeit auch die verfestigten Ungleichheitslinien zwischen den Geschlechtern im Erwerbssystem lockern. „Parallel laufend

\footnotetext{
6 An dieser Stelle kann nicht auf eine Ursachenanalyse sowie auf die zahlreichen vorliegenden Konzepte und Lösungsvorschläge zu einzelnen gleichstellungspolitischen Themen eingegangen werden (vgl. u.a. Bundesregierung 2002).
} 
werden aber die ungleichheitsrelevanten Folgen der im gegenwärtigen Erwerbssystem ausgeblendeten Reproduktionserfordernisse exponentiell aufgewertet: Wer für Betrieb und Arbeit allzeitig verfügbar ist, entscheidet sich mehr und mehr an der Frage der privaten Sorge- und Betreuungsverpflichtung und somit an der individuellen Lebensform (...). Die betriebliche geforderte moderne ,Arbeitsmonade' darf zwar männlich oder weiblich (...) sein. Was sie aber nicht darf, ist, den betrieblichen Verfügungsanspruch durch außerbetriebliche Anforderungen in Frage zu stellen" (Nickel/Lohr 2005, S. 222). Der Arbeitskraftunternehmer ist vielleicht eher weiblich - ,aber er ist ganz sicher keine Mutter“ (Voß/Weiß 2005, S. 85). Diese Frauen haben entweder keine familiären Verpflichtungen. So sind Frauen in Führungspositionen häufiger kinderlos und leben in kleineren Haushalten (Kleinert et al. 2007) oder sie übertragen sie Frauen anderer sozialer Schichten, oft Migrantinnen (vgl. Lutz in diesem Heft).

\subsection{PREKARITÄT FÜR DIE ANDEREN}

Der Umbruch in der Arbeit hat widersprüchliche Folgen. Die stärker marktorientierte Steuerung verschärft für einen anderen Teil der Arbeitnehmerinnen und Arbeitnehmer das Risiko prekärer Beschäftigungen, die „deutlich unter ein Einkommens-, Schutz- und Integrationsniveau sinken, das in der Gegenwartsgesellschaft als Standard definiert und mehrheitlich anerkannt wird“ (Dörre/Fuchs 2006, S. 3). Armut trotz Arbeit ist in Deutschland zu einer Realität geworden und betrifft Frauen besonders (Adamy 2007). 30 \% der Frauen arbeiteten 2005 zu Niedriglöhnen, gegenüber $13 \%$ der Männer (Weinkopf/ Kalina 2006). Das hat zur Folge, dass viele Frauen trotz eigener Erwerbstätigkeit nicht eigenständig ihren Lebensunterhalt sichern können.

In diesem Bereich relativiert sich auch die gleichstellungspolitische Bedeutung einer steigenden Frauenerwerbstätigkeit. Auf diesem unteren Niveau von Einkommen, mangelnder Zukunftssicherung und Entfaltung in der Arbeit dürfte für Frauen das Gleichziehen mit den Arbeits- und Lebensbedingungen von Männern kaum das erstrebenswerte Ziel sein. Gleichstellungsbestrebungen sollten sich eher auf andere Gruppen als Bezugspunkt richten - unbefristet Beschäftigte, tariflich Entlohnte. Gleiches gilt für von Erwerbsarbeit ausge- schlossene Frauen, für die das zentrale Anliegen die Überwindung ihrer Exklusion ist. Die Geschlechterfrage scheint sich hier direkter als bisher mit der sozialen Frage zu verknüpfen.

Der Zwang, jegliche Erwerbsarbeit anzunehmen, ohne auf die Vereinbarkeit mit den Familienaufgaben achten zu können, kann jedoch Frauen in besonderer Weise gegenüber Männern benachteiligen. Vereinbarkeitskonflikte in diesem Beschäftigungssegment sind bisher nicht systematisch untersucht worden. ${ }^{7}$ Infolge großer Spannungen zwischen Erwerbsnotwendigkeit und Fürsorgeverpflichtungen kann Prekarität im Lebenszusammenhang entstehen.

\subsection{NEUE FRAGEN}

Kann es sein, dass die bisherige Gleichstellungspolitik vor allem für das Segment relativ geschützter Arbeit mittlerer Qualifikation weiterhin aktuell ist, dass aber für andere Gruppen von Frauen modifizierte Konzepte erforderlich sind? Wie ist die spezifische Interessenlage in Bezug auf Gleichstellungspolitik an den „Polen“ - bei den „high potentials“ und im „Prekariat“? Dieser Frage muss für eine künftige Gleichstellungspolitik nachgegangen werden.

Dabei sieht es so aus, als ob auch Geschlechterfrage und Vereinbarkeitsproblem mit dem Umbruch in der Arbeit auf neue Weise zusammenhängen: Für die (relativen) Gewinnerinnen der gegenwärtigen Umbrüche könnte die empirisch beobachtete "Dethematisierung von Geschlecht" die Vereinbarkeitsfrage aufwerten. Diese qualifizierten, durchsetzungsstarken Frauen können bestimmte berufliche Positionen durchaus erreichen, es sei denn, ihre Fürsorgeaufgaben hindern sie an der geforderten Allzeitverfügbarkeit. Vielleicht werden sie mit dem Rückenwind des Aufschwungs familienpolitischer Rhetorik zu Vorkämpferinnen einer kulturellen Veränderung in der Arbeit im Sinne von Work-Life Balance. Vielleicht werden sie aber auch, wenn sie Kinder haben, wie bisher eher hinausgedrängt und diskriminiert.

Für die Verliererinnen könnten Konflikte zwischen Fürsorge und Erwerbstätigkeit ein Teilaspekt ihrer insgesamt prekären Lebenslage sein. Diese Frauen könnten damit verletzbarer als Männer derselben sozialen Gruppe sein. Für sie reicht weder die Lösung der Vereinbarkeitskonflikte noch die bloße Gleichstellung mit prekär be- schäftigten Männern. Doch ohne bessere Vereinbarkeitsbedingungen sind sie erst recht gefährdet. Daneben benötigen sie eine Politik der Entprekarisierung, die sich sowohl auf den sozialen Schutz ihrer Arbeitsverhältnisse als auch auf die Qualität der Arbeit selbst beziehen muss.

Auf die Probleme der ersten Gruppe hat die Politik mit der neuen Familienpolitik reagiert, die der zweiten Gruppe werden bisher kaum beachtet.

\section{1 \\ Gleichstellungspotenziale der Familienpolitik}

\subsection{GEGEN DIE GESCHLECHTS- SPEZIFISCHE ARBEITSTEILUNG}

Erklärtes Ziel der modernisierten Familienpolitik ist die Erhöhung der Frauenerwerbstätigkeit. Damit weist sie, auch wenn die Motive der Familienpolitik in erster Linie ökonomischer und demografischer Natur sind, in die gleiche Richtung wie die Gleichstellungspolitik. Letztere zielte immer auch darauf, die Möglichkeiten für Frauen zu verbessern, beruflich tätig zu sein. Frauen sollten eine befriedigende berufliche Tätigkeit ausüben können, die ihnen die Anwendung ihrer Fähigkeiten erlaubt und sie ökonomisch unabhängig macht. Sie sollten ebenso wie Männer die mit der Erwerbsarbeit verbundene soziale Einbindung und Anerkennung erlangen können. In der Teilhabe an Erwerbsarbeit ein emanzipatorisches Potenzial zu sehen, entspricht den Ansichten der übergroßen Mehrheit der Frauen. Diese Logik hat ihr Pendant in der Forderung, dass Männer stärker an der unbezahlten Arbeit in der Familie teilnehmen, die traditionell von Frauen verrichtet wird. Ein Ziel von Gleichstellungspolitik war und ist somit die Aufhebung der geschlechtsspezifischen Arbeitsteilung.

Insofern ist die Umorientierung in der Familienpolitik keineswegs gleichstellungspolitisch bedeutungslos. Bis vor Kurz-

\footnotetext{
7 Diesem Thema widmet sich ein im August 2007 begonnenes empirisches Forschungsprojekt am WSI „Flexible Familienernährerinnen. Prekarität im Lebenszusammenhang ", das von der Autorin zusammen mit Petra Drauschke durchgeführt wird.
} 
em hatte die Politik vorrangig die traditionelle Familie unterstützt und lange Erwerbsausstiege von Müttern gefördert. Wenn nun Familienpolitik die Bedingungen verbessern will, unter denen Mütter beruflich tätig sein können, und auch Anreize setzt, früh an den Arbeitsplatz zurückzukehren, geht damit ein Leitbildwechsel in der Politik einher: Die gute Mutter ist nicht mehr diejenige, die ihr Kind zuhause betreut, sondern Berufstätigkeit und Mutterschaft werden als vereinbar angesehen (vgl. Jurczyk in diesem Heft).

Der Leitbildwechsel hin zur vollen Akzeptanz der berufstätigen Mutter ist seit Langem überfällig und vollzieht lediglich nach, was für viele Frauen längst Realität ist. Wenn nun der grundsätzliche Anspruch von Frauen anerkannt wird, durch die Geburt von Kindern nicht mehr beruflich aufs Abstellgleis geschoben zu werden, und dies durch entsprechende Maßnahmen untermauert wird, so kann diese Familienpolitik einen Beitrag zur Gleichstellung der Geschlechter leisten. In diese Richtung gehen das Teilzeit- und Befristungsgesetz 2001, das Tagesbetreuungsausbaugesetz (TAG) sowie der jüngst beschlossene Ausbau der Kinderbetreuungsinfrastruktur. Die Neuregelungen zu Elternzeit und Elterngeld orientieren auf kürzere Berufsunterbrechungen und - vorsichtig - auf die Beteiligung der Väter an der Betreuung des Kindes. Mit dem neuen Elterngeld wird die Einbindung von Männern in die Fürsorgearbeit gefördert. Erstmals werden materiell fundierte Schritte getan, Väter zur Übernahme von Fürsorgearbeit gegenüber ihren kleinen Kindern zu ermutigen. Hier scheint die Familienpolitik von den Gleichstellungsdiskursen beeinflusst zu sein. Stärker noch als die für Väter reservierten Monate der Elternzeit ist dafür das Prinzip der Einkommensersatzleistung beim Elterngeld zu gewichten, da es das Argument außer Kraft setzt, dass die junge Familie sich den Einkommensausfall des oft besser verdienenden Vaters nicht leisten kann.

\subsection{LEERSTELLEN UND DEFIZITE}

Die gegenwärtige Vereinbarkeitsförderung weist aber auch wichtige Defizite auf, deren Überwindung für ihre gleichstellungspolitische Wirkung essenziell ist:

(1) Die neue Familienpolitik wird verkürzt auf die Überwindung der elementaren Hindernisse, die kurz nach der Geburt ei- nes Kindes auftreten (Einkommensausfall eines Partners, Mangel an Betreuungsmöglichkeiten für Kleinkinder). Der Dimension der notwendigen Umgestaltung zur Lösung der Fürsorgefrage, die sowohl alle Phasen im Leben eines Kindes als auch Pflegesituationen im Blick haben müsste, wird sie nicht gerecht. Von einem umfassenden Konzept, wie eine gesellschaftliche Lösung der Care-Frage aussehen kann, sind wir weit entfernt.

Insbesondere die notwendigen Umgestaltungen in den Betrieben, vor allem familiengerechte Arbeitszeiten (über Teilzeit hinaus) und Arbeitsorganisation, sind kaum Gegenstand von Politik; für sie wird gegenwärtig lediglich geworben. Konkrete Regelungen sind - mit Ausnahme einer unbezahlten Pflegefreistellung - bisher nicht vorgesehen. Freiwillige Aktivitäten werden aber vorwiegend auf die Bereiche beschränkt bleiben, in denen Betriebe um qualifiziertes Personal konkurrieren.

(2) Die Familienpolitik wirkt stark sozial differenzierend. Die größten Effekte sind für Mütter mit höherem Einkommen zu erwarten. Sie profitieren am meisten vom Einkommensersatz beim Elterngeld. Sie können auch, wenn nötig, kostspielige Kinderbetreuungsarrangements nach der Rückkehr aus der Elternzeit finanzieren, wenn die Kinderbetreuungsmöglichkeiten für Einjährige, wie zu erwarten, nicht ausreichen werden. Andere Frauen dagegen, die keinen Kinderbetreuungsplatz erhalten werden und private Lösungen nicht finanzieren können, müssen durch die Verkürzung der Bezugsdauer des Elterngeldes finanzielle Nachteile hinnehmen und weiterhin vom Partner abhängig sein. Auch unfreiwillig nicht erwerbstätige Mütter (Langzeitarbeitslose) sowie Studierende haben durch die Reform Nachteile, für die ein angemessener sozialpolitischer Ausgleich bislang noch aussteht. Generell sind Vereinbarkeitsprobleme von Frauen in prekären Arbeits- und Lebensverhältnissen unzureichend thematisiert, geschweige denn gelöst.

(3) Der Paradigmenwechsel in der Familienpolitik ist alles andere als konsequent. Elterngeld, obwohl als Lohnersatzleistung konzipiert, wird auch an nichterwerbstätige Ehefrauen gezahlt. ${ }^{8}$ Bothfeld (2006) zeigt an der unentschiedenen Ausgestaltung des Elterngeldes auf, dass das alte, der Familienpolitik zugrunde liegende Paradigma der Anerkennung von Fürsorgearbeit nicht abgelöst, sondern vom neuen Paradigma des „Integrationsmodells“ überlagert wird. Auch wird die egalitäre Lösung, bei der beide Eltern Elternzeit mit Teilzeit kombinieren, mit der neuen Regelung materiell schlechter gestellt (ebd.).

(4) Weitgehend unbeachtet ist bisher geblieben, dass sich die Vereinbarkeitsförderung losgelöst neben der großzügigen Subventionierung des traditionellen Ernährermodells vollzieht. Keine einzige der alten Regelungen - Ehegattensplitting, kostenfreie Mitversicherung der Ehefrauen in der Krankenkasse, Hinterbliebenenversorgung - wurde angetastet.

\section{Fazit}

Die Analyse hat drei Punkte deutlich gemacht:

(1) Deutschland braucht wieder eine aktive Gleichstellungspolitik, die über die Förderung der Vereinbarkeit von Familie und Beruf hinausgeht. Denn die Gleichstellung von Frauen und Männern ist bei der Förderung existenzsichernder Erwerbsarbeit, der Entgeltgleichheit und der Veränderungen der geschlechtsspezifischen Arbeitsteilung in Deutschland nur schleppend vorangekommen. Geschlechtergerechte Arbeitszeiten sind ebenso wenig erreicht wie die eigenständige Existenzsicherung von Frauen. Fragen einer mangelnden Vereinbarkeit von Familie und Beruf sind in viele Gleichstellungsprobleme mit eingeschlossen, aber oft nur Teil des Problems. Einkommensdiskriminierung von Frauen sowie von Teilzeitbeschäftigten und die verfestigte horizontale und vertikale Segregation nach Geschlecht sind nur Beispiele von Ungleichheiten, die einen umfassenden Gleichstellungsansatz erfordern.

(2) Für eine moderne Gleichstellungspolitik stellen sich einige Fragen neu. Dies hängt mit der wachsenden sozialen Diffe-

\footnotetext{
8 Eine ähnlich hybride Konstruktion ist jetzt von einigen CDU-Politikern angedacht, die im Zusammenhang des geplanten Ausbaus der Krippenplätze eine Prämie für "selbsterziehende" Hausfrauen fordern.
} 
renzierung, auch unter Frauen, zusammen. Ökonomische Umbrüche bedingen, dass sich um ein relativ geschütztes mittleres Segment von Frauenbeschäftigung herum eine Polarisierung entwickelt: Frauen mit riskanten Chancen, die tendenziell von den Umbrüchen profitieren, und Frauen auf der Verliererseite mit prekären Arbeitsund Lebensbedingungen. Gleichstellung der Geschlechter erfordert, differenzierte Politikansätze für bestimmte Gruppen zu entwickeln. Sie erfordert aber auch, dass die allgemeine Frage sozialer Ungleichheit (wieder) auf die Tagesordnung gesetzt wird. Von einer Politik der Entprekarisierung würden besonders Frauen profitieren.

An beiden Polen könnte sich der $\mathrm{Zu}$ sammenhang von Gleichstellung und Vereinbarkeitsfrage neu darstellen, indem die geforderte allzeitige betriebliche Verfügbarkeit Familienverantwortung nahezu ausschließt. Für die relativen Gewinnerinnen kann das mit beruflichen Chancen einhergehen, für die anderen kann sich die prekäre Beschäftigung zur Prekarität im Lebenszusammenhang ausweiten. Möglicherweise liegt aber im Ringen um eine Balance von Arbeit, Familie und sonstigen Aktivitäten ein verbindender Aspekt künftiger Gleichstellungspolitik.

(3) Gleichstellungspolitik und Familienpolitik weisen gegenwärtig Schnittmengen auf, wenn es um Förderung der Müttererwerbstätigkeit und väterlicher Fürsorgebeteiligung geht. Die Familienpolitik hat gleichstellungspolitische Anliegen aufgegriffen. Sie kann, soweit sie tatsächlich die Vereinbarkeit von Familie und Beruf erleichtert, einen Beitrag zur Veränderung der geschlechtsspezifischen Arbeitsteilung leisten: Sie hilft, jahrelange Berufsunterbre- chungen $\mathrm{zu}$ vermeiden, und orientiert ansatzweise auf die Familienarbeit von Vätern. Sie ist auch geeignet, das gesellschaftliche Klima zu verändern und Geschlechterstereotype abzubauen. Von der Vereinbarkeitspolitik können vielleicht auch Rückwirkungen auf die Gleichstellung in anderen Bereichen, z. B. beim Einkommen, erwartet werden, wenn Mütter künftig besser imstande sind, kontinuierlich und mit höherem zeitlichen Umfang erwerbstätig zu sein.

Insofern ist die Umorientierung der Familienpolitik gleichstellungspolitisch keineswegs bedeutungslos. Eine umfassende Gleichstellungspolitik, die auf die veränderten Bedingungen auch mit weiterentwickelten Konzepten reagieren muss, kann sie aber nicht ersetzen.

\section{LITERATUR}

Adamy, W. (2007): 1,2 Millionen können vom Arbeitseinkommen nicht leben, in: Soziale Sicherheit 5, S. 180-189

Beckmann, P. (2003): Beschäftigungsquote von Frauen - (k)ein guter Indikator für die Erwerbstätigkeit von Frauen, hrsg. vom IAB Nürnberg Berghahn, S. (2003): Der Ritt auf der Schnecke, 23.08.07: http://web. fu-berlin.de/gpo/pdf/berghahn/Ritt_auf_der_Schnecke.pdf Bothfeld, S. (2006): Das Elterngeld - Einige Anmerkungen zum Unbehagen mit der Neuregelung, in: femina politica 2, S. 102-107

Bothfeld, S./Klammer, U./Klenner, Ch./Leiber, S./Thiel, A./Ziegler, A. (2005): WSI-FrauenDatenReport 2005. Handbuch zur wirtschaftlichen und sozialen Situation von Frauen, Berlin

Brader, D./Lewerenz, J. (2006): Frauen in Führungspositionen. An der Spitze ist die Luft dünn, IAB-Kurzbericht 2

Bundesregierung (2002): Bericht der Bundesregierung zur Berufs- und Einkommenssituation von Frauen und Männern, erstellt vom WSI in der Hans-Böckler-Stiftung, INIFES, Forschungsgruppe Tondorf; Bundestagsdrucksache 14/8952

Bundesregierung (Hrsg.) (2006) 2. Bilanz Chancengleichheit, Frauen in Führungspositionen, Berlin

Dörre, K./Fuchs, T. (2006): Prekarität und soziale (Des-)Integration, in: Z. (Zeitschrift Marxistische Erneuerung), http://www.linksnet.de/ drucksicht.php?id=1940
EU-Kommission (2007): Bericht der Kommission an den Rat, das europäische Parlament, den europäischen Wirtschafts- und Sozialausschuss und den Ausschuss der Regionen zur Gleichstellung von Frauen und Männern 2007, Brüssel

Funke, C./Suder, K. (2007): A Wake-Up Call for Female Leadership in Europe, Paper von McKinsey für den Global Summit of Women, Berlin Gottschall, K. (2000): Soziale Ungleichheit und Geschlecht, Opladen Habermann-Horstmeier, L. (2007): Karrierehindernisse für Frauen in Führungspositionen. Ergebnisse einer empirischen Studie an 300 Frauen aus dem deutschen Mittel- und Topmanagement, Schriftenreihe Band I des Steinbeis Technologietransferzentrums, Villingen-Schwenningen Holst, E. (2007): Arbeitszeitwünsche von Frauen und Männern liegen näher beieinander als tatsächliche Arbeitszeiten, in: DIW-Wochenbericht 14-15, S. 209-215

Holst, E./ Schrooten, M. (2006): Führungspositionen: Frauen geringer entlohnt und nach wie vor seltener vertreten. in: DIW-Wochenbericht 25, S. 365-372

Holst, E./Stahn, A.-K. (2007): Zu wenige Frauen in Spitzenpositionen in großen Banken und Versicherungen, in: DIW-Wochenbericht 26, S. 405407

Institut für Demoskopie Allensbach (2006): Generationenbarometer 2006, (IFD-Umfrage 4297), Freiburg/München, S. 302 
Jurczak, K./Hurley, J. (2007): "Mind the gap" - Quality of work for women and men, in: Foundations Focus, European Foundation for the Improvement of Living and Working Conditions, S. 6-9

Jurczyk, K./Voß, G. (2000). Entgrenzte Arbeitszeit - reflexive Alltagszeit, in: Hildebrandt, E./Linne, G.: Reflexive Lebensführung. Zu den sozialökologischen Folgen flexibler Arbeit, Berlin, S. 151-205

Klammer, U. (2007): Zeit und Geld im Lebensverlauf - Empirische Evidenz und sozialpolitischer Handlungsbedarf aus der Geschlechterperspektive, in: Intervention 1, S. 145-174

Kleinert, C./ Kohaut, S./ Brader, D./ Lewerenz, J. (2007): Frauen an der Spitze. Arbeitsbedingungen und Lebenslagen weiblicher Führungskräfte, Frankfurt/New York

Klenner, Ch./ Pfahl, S. (2007): Zwischen Zeitnot und Karriereverzicht, in Heitkötter et al. (Hrsg.): Zeit für Beziehungen? Zeit in und Zeitpolitik für Familien (im Erscheinen)

Kratzer, N./ Fuchs, T./ Wagner, A./ Sauer, D. (2005): Zeitmuster - Zeitverwendung im Kontext von Erwerbsarbeit und Haushalt, in: SOFI/IAB/ISF/INIFES (Hrsg.): Berichterstattung zur sozioökonomischen Entwicklung in Deutschland. Arbeit und Lebensweisen, Erster Bericht, Wiesbaden, S. 381-402
Lohr, K./Nickel, H. M. (2005): Subjektivierung von Arbeit - Riskante Chancen, in: Dies. (Hrsg.): Subjektivierung von Arbeit - Riskante Chancen, Münster, S. 207-239

OECD (2001) Employment Outlook June

Sauer, D. (2005): Arbeit unter (Markt-)Druck: Ist noch Raum für innovative Arbeitspolitik?, in: WSI-Mitteilungen 4, S. 179-185

Statistisches Bundesamt (2007): Pressemitteilung vom 19.07.

Statistisches Bundesamt (Hrsg.) in Zusammenarbeit mit WZB und ZUMA (2006): Datenreport 2006. Zahlen und Fakten über die Bundesrepublik Deutschland

Voß, G./ Weiß, C. (2005): Ist der Arbeitskraftunternehmer weiblich? in: Lohr, K./Nickel, H. M.: Subjektivierung von Arbeit - Riskante Chancen, Münster, S. 65-91

Wanger, S. (2005): Frauen am Arbeitsmarkt. Beschäftigungsgewinne sind nur die halbe Wahrheit, IAB-Kurzbericht 22

Weinkopf, C. /Kalina, Th. (2006): Mindestens sechs Millionen Niedriglohnbeschäftigte in Deutschland: Welche Rollen spielen Teilzeitbeschäftigung und Minijobs?, IAT-Report 2006-03

WSI-Frauenlohnspiegel (o. J.) www.frauenlohnspiegel.de sowie http://www.boeckler.de/cps/rde/xchg/SID-3D0AB75D-96BC1202/hbs/ hs.xsl/320_85262.html (12.7.07) 